Recording the results by age-groups for each sex (see Table) showed a higher proportion of normals for females, and this is most marked for the age-group of 11 to 20 years. In this last group almost two-thirds of the appendices removed were judged to be normal, whereas only a quarter of those from males of the same age-group were so judged.

In most, if not all, of these patients a wrong diagnosis of appendicitis had been made. Such errors occur at all ages and in both sexes, but they are much commoner in young females. In this last group the symptoms that are mistaken for those of appendicitis are not uncommonly derived from the ovary-for example, they are sometimes related to rupture of a follicle. In many cases I believe that they have a large psychological element-leaving the smoother life of school to start work, having a job that is not liked, quarrelling with a boy friend, and other emotional upsets commoner at this age than at others. It is, however, outside my province to determine the reasons for these mistakes in diagnosis: I wish to draw attention to the frequency of error in this group so that clinicians may be especially wary when making a diagnosis of acute appendicitis in young females.

\section{Summary}

A mistaken diagnosis of acute appendicitis is made much more often in females aged 11 to 20 years than in any other age-group. Figures are given to show that as many as two out of three appendices removed from this group may be quite normal.

\section{REFERENCES}

Barnes, B. A., Behringer, G. E., Wheelock, F. C., and Wilkins, E. W. (1962). J. Amer. med. Ass., 180, 122. Lee, J. A. H. (1961). Lancet, 2,815 .

\title{
TWO CASES OF THROMBOEMBOLIC DISEASE ASSOCIATED WITH ORAL CONTRACEPTIVES
}

BY

\author{
N. MCINTYRE, M.B., B.S., B.Sc. \\ M. J. PHILliPS, M.B., Ch.B., D.C.P.
} AND

\section{J. C. VOIGT, B.M., B.Ch., D.Obst.R.C.O.G.}

Flight Lieutenants, Royal Air Force Hospital, Steamer Point, Aden

The annotation in the British Medical Journal of August 4 (p. 315) on the possible dangers of oral contraceptives has prompted us to submit without delay these two case reports of thrombotic disease associated with the taking of tablets containing norethynodrel. For climatic reasons many women wish to avoid pregnancy in the tropics, and since "conovid" can be bought without restriction from local chemists' shops it is likely that many women are availing themselves of the advantages of oral contraception. The second patient took "enavid" as treatment for menstrual disorder, and, in common with the cases mentioned in your annotation, she has had previous thrombotic disease following pregnancy. The first patient, however, has no previous history of thromboembolic disease.

\section{Case 1}

A 28-year-old married woman with two children was admitted to R.A.F. Hospital, Aden, on August 6.1962. For two years she had suffered from attacks of indigestion lasting three days to three weeks. Each attack was associated with epigastric discomfort, an ache posteriorly over the right lower ribs. a choking sensation, and vomiting of a clear fluid. Appetite at these times was poor, but originally food seemed to relieve the discomfort. For some years she had taken large doses of purgatives, making her stool watery. After arrival in Aden six months ago her abdominal symptoms were exacerbated and she was admitted to hospital on June 6 for investigation. Physical examination revealed no abnormality. Her hasmoglobin was 12 g. $/ 100 \mathrm{ml}$.; E.S.R. (Westergren) was $6 \mathrm{~mm}$./hr.; W.B.C. was $6.000 / \mathrm{c} . \mathrm{mm}$. with normal differential. Chest $x$-ray and urine examinations were normal. No faecal occult blood was found. Frac- tional test meal showed a high acid curve. She was discharged on June 18 and a barium-meal examination done as an out-patient on July 6 was normal. She was treated with antacids and her symptoms improved.

On August 3 the gradual onset of a pleuritic pain occurred over the lower part of the left lung anteriorly. with haemoptysis of considerable amounts of dark-red blood with accompanying clear sputum. Chest $x$-ray examination showed no change from the normal picture of June 8 . Pleurisy and haemoptysis persisted until her admission on August 6, with associated breathlessness due to pain. She had no other symptoms on systemic review. Her weight had been steady and appetite good. Periods had been regular with a 28-day cycle and lasting seven days. She was a premature baby, and had rheumatic fever as a child. She has two children, aged 8 and $4 \frac{1}{2}$; both pregnancies were normal, with no complications and no thrombotic phenomena. Her mother has angina and her father has "ulcers." There was no other relevant family history. She smokes 15 cigarettes a day but drinks very little alcohol.

On admission she looked well but tired, and her temperature was $99.6^{\circ} \mathrm{F}$. $\left(37.6^{\circ} \mathrm{C}\right.$.). There was no anaemia, cyanosis, dyspnoea, dehydration, jaundice, or lymphadenopathy. Hess's test was negative. and there was no evidence of thrombosis of any limb vein. Rectal examination revealed no pelvic tenderness or cause for thrombosis. Pulse was regular at $100 / \mathrm{min}$. and blood-pressure $135 / 85$. Her jugular pressure was not raised and there was no oedema. Her heart was not enlarged, and the cardiac impulse was normal. The pulmonary second sound was not accentuated, and there were no murmurs before or after exercise. The trachea was central, and, apart from a transient rub at the left base and painful restriction of left chest movement, there were no unusual physical signs. The abdomen was normal, and neurological examination revealed no abnormality.

Her haemoglobin was 12.3 g./100 ml., E.S.R. $45 \mathrm{~mm}$. $/ \mathrm{hr}$.. and W.B.C. 7,800 (P. $66 \%$, L. $21 \%$, M. $6 \%$. E. $7 \%$ ). Chest $x$-ray examination on admission showed some coarse horizontal streaking in the right costophrenic angle and possibly some linear streaking near the left costophrenic angle. Sputum was heavily blood-stained. but there were no acidalcohol fast bacilli or pus cells on direct examination and no growth on culture. Urine was normal. Heaf test was negative. No L.E. cells were found. Other investigations revealed serum proteins $6.8 \mathrm{~g} . / 100 \mathrm{ml}$. with $\mathrm{A}: \mathrm{G}$ ratio $1.8: 1$. Blood group $\mathrm{AB}$ Rh-negative, clotting-time $8 \mathrm{~min} .20 \mathrm{sec}$. (Lee and White), bleeding-time $3 \mathrm{~min} .25 \mathrm{sec}$. (Duke), and prothrombin time normal. Serum glutamic-oxaloacetic transaminase was 56 Sigma-Frankel units $/ \mathrm{ml}$. on August 14 . Other liver-function tests were normal.

The most likely diagnoses appeared to be pulmonary infarction. tuberculosis. bronchial adenoma, cr an atypicitl 
pneumonia. She felt well, and it was decided to delay specific therapy and to give mild analgesics for the pain while waiting for the results of laboratory tests.

Frank haemoptysis continued, and the pleuritic pain became worse and was associated with an aching in the left shoulder and arm. On August 10 she had been apyrexial for four days and there was no clinical evidence of right ventricular strain. There was impaired percussion at the left base with crepitations and rhonchi. Chest $x$-ray examination showed clearing at the right costophrenic angle but elevation of the left side of the diaphragm and shadowing in the posterior recess in the lateral film. E.C.G. showed slight right axis deviation and a vertical heart with a small $Q$ and flat $\mathrm{T}$ waves in lead III. There was no clockwise rotation and no evidence of right ventricular strain in the precordial leads. The E.S.R. remained at $42 \mathrm{~mm}$./ hr., but the W.B.C. had risen to $14,300 /$ c.mm., with $80 \%$ polymorphs.

On August 11 the temperature rose to over $100^{\circ} \mathrm{F}$. $\left(37.8^{\circ} \mathrm{C}.\right)$, and she felt unwell. The pulse rate had risen to $120 /$ min., but there was still no evidence of right ventricular embarrassment and the jugular venous pressure was not raised. There was gastric resonance to a higher level than normal in the left axilla and diminished breath sounds but no adventitiae at the left base.

At this stage pulmonary infarction seemed to be the most likely cause for the pleurisy and haemoptysis. However, there was no obvious source for pulmonary emboli and no suggestion of predisposing factors in her past history. We were reluctant to commence treatment with anticoagulants in view of her frank haemoptysis and uncommon blood group. and we did not feel we could exclude tuberculosis or another inflammatory lesion with certainty. Therefore large doses of crystalline penicillin were given with streptomycin, P.A.S., and isoniazid.

Following initiation of chemotherapy there was continued rise of temperature, and on August 13 pleuritic pain began at the right base. This continued while the left-sided pain cleared. Next day vaginal bleeding occurred although a normal period had finished only one week before. There was increase in the loudness of the pulmonary second sound and there were poor breath sounds at both bases, with numerous crepitations on the right side. The E.S.R. had risen to $93 \mathrm{~mm}$./hr., W.B.C. was $14,800 / \mathrm{c} . \mathrm{mm}$., with $90 \%$ polymorphs. Radiologically there were new opacities at both bases, and the left diaphragm was still raised. The E.C.G. showed lower voltage in all leads with flattening of $\mathrm{T}$ waves in all precordial leads.

Because of the fresh vaginal bleeding and the possibility of a pelvic source of pulmonary emboli, gynaecological opinion was sought. Direct questioning led to the discovery that she had recently completed one month's course of conovid for contraceptive purposes. These had been bought locally over the counter, and several of her friends were also taking them. Pelvic examination revealed a slightly bulky globular uterus and a palpable left ovary.

In vicw of recent newspaper publicity on thromboembolic disease associated with conovid, we felt that a similar relationship could not be excluded in this case. Antituberculous chemotherapy was stopped and treatment with heparin. 12.500 units intramuscularly every 12 hours, initiated. The effect of the heparin was checked by estimation of coagulation time. It was decided that if no marked exacerbation of haemorrhage from lung or uterus occurred, then phenindione could be used to continue her anticoagulant therapy. During the first 36 hours of heparin therapy the temperature fell from $103^{\circ} \mathrm{F}$. $\left(39.4^{\circ} \mathrm{C}\right.$.) to under $100^{\circ} \mathrm{F}$. $\left(37.8^{\circ} \mathrm{C}\right.$.). but climbed again to $102^{\circ} \mathrm{F}$. $\left(38.9^{\circ} \mathrm{C}\right.$. $)$ on the evening of August 17. Phenindione was started on August 19, and there was a gradual fall in temperature unti? on August 21 it was normal for the first time in 11 days. Continuous improvement seems to be occurring at the time of writing.

\section{Case 2}

A 27-year-old married woman was admitted to hospital on August 15, 1962. On May 16 she started a course of oral enavid, $5 \mathrm{mg}$. daily, on days 5 to 25 of each menstrual cycle. Three cycles were completed on this regime, and the last dose was taken on August 3. Enavid was prescribed for symptoms of premenstrual tension associated with oligomenorrhoea in the absence of any abnormality on gynaecological examination.

When 12 years old she had St. Vitus's dance and rheumatic fever, apparently with cardiac involvement. During her 16th year she suffered an attack of jaundice and later an illness associated with swelling of the face, feet, and hands. but without haematuria. This was diagnosed as acute nephritis. She had had three normal pregnancies and one incomplete abortion at 12 weeks. Urine analysis in all her pregnancies was normal.

Her last pregnancy, in 1959, was complicated by late puerperal thrombophlebitis of the left leg necessitating six weeks in hospital. This condition had recurred on two occasions since, unassociated with pregnancy. Since the miscarriage, which was in 1957, her menstrual loss steadily diminished until it was lasting only one day, although the cycle remained regular at 28 days. In the week before and the week after each period she complained of dull, aching lower abdominal pain without backache, but accompanied by irritability and a sensation of abdominal distension in the premenstrual period. It was for these complaints that enavid was prescribed. Her menstrual loss became even more scanty on treatment, but the abdominal distension. which increased during the first two treated cycles. subsided in the third, and except for slight breast tenderness she claimed subjective improvement.

Eight days prior to the present admission she experienced a cramp-like pain of four hours' duration in the left calf which recurred the next day. The left calf was swollen. tender, and gave a positive Homan sign. She was treated at home with bed rest and given oral phenylbutazone, but was always apyrexial. Next day she developed swelling and blue skin discoloration around her right ankle. This disappeared spontaneously.

On three occasions in the five days prior to admission she experienced episodes of acute chest pain, which was initially stabbing and then aching in character. The attacks were of five minutes' duration and were accompanied by breathlessness. The day before admission she developed pain and tenderness in the medial aspect of the left thigh.

On admission she looked well, was apyrexial with a sinus tachycardia of 110 a minute. She was tender over the ankle, popliteal fossa, and along the line of the long saphenous vein of the left thigh, which was $\frac{1}{2}$ in. $(1.3 \mathrm{~cm}$.) greater in circumference than the right thigh. There was no redness or warmth of the overlying skin. The B.P. was $13^{\prime} 5 / 85 \mathrm{~mm}$. $\mathrm{Hg}$ and there was no clinical evidence of rheumatic heart disease or right ventricular embarrassment. Examination of the respiratory and alimentary systems revealed no abnormalities. A clinical diagnosis of deep-vein thrombophlebitis of the left leg was made and treatment with phenindione instituted. Laboratory investigations showed: $\mathrm{Hb} 12 \mathrm{~g} . / 100 \mathrm{ml}$., E.S.R. $42 \mathrm{~mm}$. in the first hour. W.B.C. $15,600 /$ c.mm., $86 \%$ polymorphs. Platelet count 250,$000 ;$ c.mm. The S.G.O.T. was 44 Sigma-Frankel units $/ \mathrm{ml}$. Coagulation and bleeding-times were within the normal range. A chest $x$-ray examination was normal. E.C.G. was normal. At the time of writing (August 21. 1962) the patient was well and no further complications had occurred.

\section{Discussion}

In Aden it is possible to buy oral contraceptive products over the counter without a doctor's prescription, and our information suggests that quite a few women are now buying one or other of the oral contraceptives.

It is perhaps worth drawing attention to the fact that both patients had a history of rheumatic disease. It is conceivable that a combination of factors is necessary 
for the production of a thromboembolic disorder as a side-effect of oral contraceptive drugs and one of these factors might be a primary " endothelial defect." This is conjecture, but these two cases underline the need for careful observation of women using oral contraceptive drugs.

However, we would emphasize that in the two cases reported we are merely drawing attention to thromboembolic disease cccurring in association with the use of oral contraceptive drugs-without suggesting any definite causal relationship.

\section{Summary}

Two cases are reported of thrombotic or thromboembolic disease occurring in women who had been taking oral contraceptive drugs. The first case presented as haemoptysis and pleurisy. The clinical and radiological course was one of multiple pulmonary infarcts responding to anticoagulant therapy after a severe and potentially fatal illness. There was no obvious peripheral source for emboli and no previous history of thrombophlebitis. She had taken "conovid" for one month for contraceptive purposes. Case 2 had a thrombophlebitis in the veins of the left leg with several short episodes of chest pain and dyspnoea suggestive of pulmonary embolism. She had a history of previous attacks of thrombophlebitis following late puerperal venous thrombosis in the left leg in 1959. She had been given " enavid" for menstrual disturbance. The only other feature common to both cases was that they had suffered from rheumatic fever in childhood, but there was no evidence of cardiac sequelae.

ADDENDUM.-Since the above case histories were written both women have been discharged from hospital. Case 1 had developed a localized effusion at the right base before discharge. It extended up into the greater fissure and is slowly clearing. Case 2 now has a mild recurrence of thrombophlebitis in the left leg 25 days after cessation of anticoagulant therapy on September 6 .

We thank the Director-General of Medical Services, Royal Air Force, for permission to publish details of these cases.

\section{Preliminary Communications}

\section{Autoimmune Antibodies in Infantile Cirrhosis}

Cirrhosis of the liver in early childhood is very common in India. Hardly a day passes when one does not see a few cases in the out-patient department. The age of onset is 1 to 3 years. The clinical symptoms during the early stages of infantile cirrhosis are vagueusually gastro-intestinal disturbances like diarrhoea, flatulence, and distension of the abdomen. The liver soon becomes palpable, with a sharp edge. As the disease advances the liver becomes firmer, the spleen enlarges, subcutaneous abdominal veins become dilated, and ascites and jaundice appear. According to Achar (1955), approximately $60 \%$ of the infants seen in his clinic died between three months and three years after the onset of the disease, $10 \%$ survived with a stabilized condition, and $30 \%$ recovered.

Several possible causes have been suggested-for example, stenosis of the small bile-ducts leading to the biliary cirrhosis (Gibbons, 1890 ; Lahiri, 1936), veno- occlusive disease (Bras et al., 1954 ; Rao, 1934, 1935), bacterial infection (Lahiri, 1936), and viral infection (Narayanamurthi et al., 1939 ; Kutumbiah, 1956). The familial occurrence of the disease is ascribed to the possible transmission of the virus of hepatitis from a silent carrier (mother) to infants (Achar and Raju, 1951; Srivastava and Aikat, 1954).

Dietary factors have always been suspected to be the cause of infantile cirrhosis; there is, however, no agreement among the observers regarding the exact mode involved. Overfeeding (Mukherjee, 1922), excess of starch intake (Gibbons, 1890), vitamin deficiencies (Green-Armytage, 1926), feeding of mother's milk (Narayanamurthi et al., 1939), and cow's milk (Rao, 1950) have been blamed for the disease. The occurrence of infantile cirrhosis among members of families in the upper and middle classes is cited against the probability of a dietary origin, contrasting it with kwashiorkor, which is more often found among members of families of the poorest section of the population (Smetana et al., 1961).

The present work was done with a view to evaluating the role of autoimmunity in the causation of the disease.

\section{Materials AND Methods}

The twelve cases studied were in the Hospital for Children, Patna. Diagnosis was made by clinical examination and by liver biopsy.

Auto-antibodies were demonstrated by tanned-red-cell agglutination (T.R.C.) test as modified by Roitt and Doniach (1958). Antigen was prepared from liver tissue obtained from cases undergoing abdominal surgery. The tissue obtained was washed with buffered saline $(p H 7)$. It was then disrupted in 4 volumes of buffered saline and centrifuged at 3,000 r.p.m. for 15 minutes. The supernatant was stored in a number of small tubes at $-15^{\circ} \mathrm{C}$. Antigens from intestinal mucosa, kidney, and thyroid were prepared in the same way to test the specificity of the antibodies.

Human group-O cells were used within two days of drawing into citrate saline solution. The cells were washed three times with phosphate buffer $p \mathrm{H} 7.2$ and made up to a $4 \%$ cell suspension. The cells were mixed with an equal volume of fresh tannic-acid solution ( $5 \mathrm{mg} . / 100 \mathrm{ml}$. buffer) and allowed to stand at room temperature for 10 minutes with occasional shaking. The tanned cells were then centrifuged gently, washed in buffer once or twice, and mixed with an equal volume of the tissue extract (liver-tissue extract for the test and others for control). This was left for about 30 minutes with occasional shaking. These antigen-coated cells were then washed carefully three times with buffer containing $1 \%$ human serum (inactivated at $56^{\circ} \mathrm{C}$. for 30 minutes) and made up to a $1 \%$ cell suspension in $1 \%$ serum buffer. Patient's serum was then taken and inactivated at $56^{\circ} \mathrm{C}$. for 30 minutes. Serial serum dilutions were made using the buffer. The dilutions were $1: 5 ; 1: 25 ; 1: 250 ; 1: 2,500 ;$ and $1: 25,000$. Then $0.1 \mathrm{ml}$. was taken on grooved slides. On the sixth slide was taken $1: 5$ serum and the liver-tissue extract to serve as an antigen inhibition control. To each slide was added $0.1 \mathrm{ml}$. of the tanned coated red cells. After thorough mixing the slides were covered and allowed to stand for two to three hours at room temperature and then overnight at $4^{\circ} \mathrm{C}$. before tests were read. The endpoint was taken as the highest serum dilution giving an even carpet of cells over the bottom of the grooves and the titre was expressed as the reciprocal of this dilution. 